\title{
A NOTE ON THE SINGULAR MANIFOLDS OF A DIFFERENCE POLYNOMIAL
}

\author{
RICHARD M. COHN
}

1. Introduction. In a previous paper ${ }^{1}$ we defined essential singular manifolds of a difference polynomial in one unknown, and gave an example of such a manifold. By an obvious extension of this definition we may say that if $A$ is an algebraically irreducible difference polynomial in unknowns $y_{1}, \cdots, y_{n}$, then an essential irreducible manifold of $A$ which annuls a polynomial of lower effective ${ }^{2}$ order than $A$ in $y_{k}, 1 \leqq k \leqq n$, or free of $y_{k}$ is an essential singular manifold of $A$ relative to $y_{k}$. The remaining essential irreducible manifolds of $A$ we shall call, as in the case of a polynomial in one unknown, ordinary manifolds relative to $y_{k}$, and the totality of solutions in these manifolds the general solution of $A$ relative to $y_{k}$,

The analogous situation in the theory of algebraic differential equations $^{3}$ suggests that the essential singular manifolds of a difference polynomial relative to one unknown are also essential singular manifolds relative to any other unknown. It is the purpose of this paper to show that this is actually the case. It will follow that we may drop the term "relative" from the concepts we have just defined. The essential irreducible manifolds of an algebraically irreducible difference polynomial may be divided into two classes, singular manifolds and ordinary manifolds. The singular manifolds are, in the sense defined above, singular relative to each unknown present in the difference polynomial. The ordinary manifolds are ordinary relative to each unknown, and the totality of solutions they contain may be called the general solution of the difference polynomial.

We make use, as in the theory of algebraic differential equations, of the separants ${ }^{4}$ of a difference polynomial. Let $A$ be a difference

Received by the editors December 18, 1947.

1 Manifolds of difference polynomials, Trans. Amer. Math. Soc. vol. 64 (1948) pp. 133-172, referred to below as M.D.P.; \$21.

2 The effective order of a difference polynomial in $y_{k}$ is defined in M.D.P. as the difference between the orders of the highest and lowest transforms of $y_{k}$ appearing effectively in the polynomial.

${ }^{3} \mathrm{~J}$. F. Ritt, Differential equations from the algebraic standpoint, Amer. Math. Soc. Colloquium Publications, vol. 14, 1932, p. 24.

4 The perhaps unexpected fact that the separant plays a rôle in the theory of difference equations was observed by Poisson, Mémoire sur les solutions particulières des equations differentielles et des équations aux différences, J. École Polytech. vol. 6 (1806) pp. 60-125. Poisson's "particular solutions" do not necessarily lie in essential singular manifolds. 
polynomial in $y_{1}, \cdots, y_{n}$. The $y_{k}$-separant of $A$ is defined as the formal partial derivative $\partial A / \partial y_{k r_{k}}$, where $r_{k}$ is the order of $A$ in $y_{k}$. It will be easy to derive the result stated above from the following theorem.

THEOREM. Let $A$ be a difference polynomial in unknowns $y_{1}, \cdots, y_{n}$. An essential irreducible manifold of $A$ which is singular relative to $y_{k}$ annuls the $y_{k}$-separant of $A$.

We proceed to prove this theorem.

2. A lemma on algebraic polynomials. We shall need solutions of difference polynomials in the form of formal power series expansions. We shall first state and prove a lemma concerning the existence of such solutions for algebraic ${ }^{5}$ polynomials.

Lemma. Let $P$ be an algebraic polynomial in unknowns $x_{1}, \cdots, x_{n}$, $z$ with coefficients in an algebraic field $K$. Let $P$ vanish, and its formal partial derivative $\partial P / \partial z$ not vanish, when $x_{1}, \cdots, x_{n}, z$ are set equal to zero. Then there exists a formal series in positive integral powers of $x_{1}, \cdots, x_{n}$ with coefficients in $K$ which annuls $P$ formally when it is substituted for $z$.

We write down the equations which result from the formal differentiation of $P=0$, when $z$ is considered as a function of $x_{1}, \cdots, x_{n}$.

$$
\begin{gathered}
\frac{\partial P}{\partial z} \frac{\partial z}{\partial x_{1}}+\frac{\partial P}{\partial x_{1}}=0, \quad \frac{\partial P}{\partial z} \frac{\partial z}{\partial x_{2}}+\frac{\partial P}{\partial x_{2}}=0, \\
\cdots \cdot \cdot, \\
\frac{\partial P}{\partial z} \frac{\partial z}{\partial x_{n}}+\frac{\partial P}{\partial x_{n}}=0 .
\end{gathered}
$$

When we substitute zero for $x_{1}, \cdots, x_{n}, z$ in these equations they determine, since $\partial P / \partial z$ does not vanish, values for $\partial z / \partial x_{i}, i=1, \cdots, n$. These values lie in $K$.

We differentiate formally the equations (1) obtaining a system of equations of the form

$$
\begin{aligned}
& \frac{\partial P}{\partial z} \frac{\partial^{2} z}{\partial x_{j} \partial x_{i}}+ \frac{\partial^{2} P}{\partial x_{j} \partial z} \frac{\partial z}{\partial x_{i}}+\frac{\partial^{2} P}{\partial x_{j} \partial x_{i}} \\
&+\frac{\partial z}{\partial x_{j}}\left(\frac{\partial^{2} P}{\partial z^{2}} \frac{\partial z}{\partial x_{i}}+\frac{\partial^{2} P}{\partial z \partial x_{i}}\right)=0, \quad 1 \leqq i, j \leqq n ; i \leqq j .
\end{aligned}
$$

${ }^{5}$ That is, polynomials in the usual sense, not difference polynomials. 
Letting $x_{1}, \cdots, x_{n}, z$ be zero in these equations, and giving the partial derivatives $\partial z / \partial x_{i}$ the values obtained in (1), we obtain unique values lying in $K$ for each $\partial^{2} z / \partial x_{i}^{2}$ and $\partial^{2} z / \partial x_{i} \partial x_{j}, i<j$.

We obtain further equations by differentiating the equations of (2). We differentiate each equation of (2) once with respect to each $x_{i}$ and retain one equation from each set of equations which differ only in the order of taking the derivatives. These equations we call (3). We treat the system (3) as (2) was treated forming (4). We continue this process and consider the system $\lambda$ consisting of the systems (1), (2), (3), ...

From the equations (3) we obtain values of the derivatives of $z$ of third order by letting $x_{1}, \cdots, x_{n}, z$ be zero and assigning to derivatives of first and second order the values already computed. In this way we obtain the derivatives of $z$ of all orders successively from the equations of $\lambda$. Evidently these will all be elements of $K$.

Using the computed values of the partial derivatives of $z$ we form the Taylor's series $z^{\prime}$ for $z$ in powers of the $x_{i}$. We shall show that this series has the properties stated in the lemma.

It is only necessary to prove that $z^{\prime}$ annuls $P$ formally when substituted for $z$. This will follow if we can show that, for every $m$, when $z$ is replaced in $P$ by the polynomial $z_{m}$ consisting of all terms of $z^{\prime}$ of degree less than $m$, the resulting polynomial $P\left(z_{m}\right)$ has no terms of degree less than $m$ in the $x_{i}$. But this in turn is equivalent to showing that, if we take the formal partial derivatives of $P\left(z_{m}\right)$ with respect to the $x_{i}$, all derivatives of order less than $m$, as well as $P\left(z_{m}\right)$ itself, vanish when the $x_{i}$ are set equal to zero. Now the equations expressing this condition are obtained from the equation $P=0$ and those equations of the system $\lambda$ which involve no derivatives of order exceeding $m-1$ by replacing $z$ by $z_{m}$ and setting $x_{1}, \cdots, x_{n}$ equal to zero. But these equations must be valid, for the partial derivatives of $z_{m}$ of order less than $m$ are identical, when the $x_{i}$ are zero, with the partial derivatives of $z^{\prime}$; and the Taylor's series $z^{\prime}$ was so constructed that its derivatives satisfy the equations $\lambda$ and $P=0$ under the stated condition. Thus the lemma is proved.

3. Proof of the theorem. We turn now to the proof of the theorem on separants. We consider an algebraically irreducible difference polynomial $A$ with coefficients in a difference field $\mathcal{~}$. The unknowns in $A$ we shall now denote, for convenience, by the letters $u_{1}, \cdots, u_{q} ; y$. We consider an irreducible manifold $\mathfrak{M}$ annulling $A$, but not $\partial A / \partial y_{r}$, where $r$ is the order of $A$ in $y$. We shall prove that $\mathfrak{M}$ is contained in or coincides with one of the ordinary manifolds of $A$ relative to $y$. 
We may assume that the order and effective order of $A$ in $y$ are equal. For, if not, we could replace $y$ by a new variable $\bar{y}=y_{k}$, where $k$ is the difference between the order and effective order. $A$ would thus be transformed into a polynomial of equal order and effective order in $\bar{y}$; and the other conditions of the problem remain unaltered.

We denote by $\Lambda$ the reflexive prime ideal consisting of all polynomials which vanish for all solutions in $\mathfrak{M}$. Let $y=\alpha, u_{i}=\gamma_{i}$, be a general point of $\Lambda$. Upon making the substitution $y=z+\alpha, u_{i}=v_{i}+\gamma_{i}$, $A$ becomes a difference polynomial $\bar{A}$ in the $v_{i}$ and $z$, which vanishes when $z$ and the $v_{i}$ are set equal to zero; while $\partial A / \partial y_{r}$ is carried into the polynomial $\partial \bar{A} / \partial z_{r}$ which does not vanish with $z$ and the $v_{i}$. It follows from the lemma that $\bar{A}$ can be annulled formally by substituting for $z_{r}$ a series $z_{r}^{\prime}$ in positive integral powers of $z, z_{1}, \cdots, z_{r-1}$, and certain $v_{i j}$. The coefficients in $z_{r}^{\prime}$ lie in the field $G$ obtained by adjoining $\alpha$ and the $\gamma_{i}$ to $\mathcal{F}$.

We now construct a formal powers series from $z_{r}^{\prime}$ by the following procedure. First we replace each coefficient by its transform in $G$. Next we replace $z_{i}$ by $z_{i+1}, i=0,1, \cdots, r-1$, throughout the series. Finally we replace each $v_{i j}$ by $v_{i, j+1}$. The resulting series we shall call $z_{r+1}^{\prime \prime}$. We substitute for $z_{r}$ in the series $z_{r+1}^{\prime \prime}$ the series $z_{r}^{\prime}$. We obtain thus a series $z_{r+1}^{\prime}$ in positive integral powers of $z, z_{1}, \cdots, z_{r-1}$ and certain $v_{i j}$, with coefficients in $G$.

Let the series $z_{r+2}^{\prime \prime}$ result from $z_{r+1}^{\prime}$ by replacing each coefficient by its transform, each $v_{i j}$ by $v_{i, j+1}$, and then replacing each $z_{i}, 0 \leqq i \leqq r-1$, by $z_{i+1}$. From $z_{r+2}^{\prime \prime}$ we obtain $z_{r+2}^{\prime}$ by replacing $z_{r}$ in the expansion by $\boldsymbol{z}_{r}^{\prime}$. We continue in this way to construct series $z_{i}^{\prime}, i=r, r+1$, $r+2, \cdots$, in powers of $z, z_{1}, \cdots, z_{r-1}$ and the $v_{i j}$, and series $z_{i}^{\prime \prime}$, $i=r+1, r+2, r+3, \cdots$ in powers of $z_{1}, z_{2}, \cdots, z_{r}$ and the $v_{i j}$. The coefficients of each $z_{i}^{\prime}$ and $z_{i}^{\prime \prime}$ lie in $G$.

Let $y_{i}^{\prime}=z_{i}+\alpha_{i}, 0 \leqq i<r$, and $y_{i}^{\prime}=z_{i}^{\prime}+\alpha_{i}, i \geqq r$. Let $y_{i}^{\prime \prime}=z_{i}+\alpha_{i}$, $1 \leqq i<r+1$, and $y_{i}^{\prime \prime}=z_{i}^{\prime \prime}+\alpha_{i}, i \geqq r+1$. Finally let $u_{i j}^{\prime}=u_{i j}^{\prime \prime}=v_{i j}+\gamma_{i j}$, $1 \leqq i \leqq q, j \geqq 0$.

We consider any polynomial $C$ in the unknowns $u_{1}, \cdots, u_{q} ; y$ with coefficients in the field $\mathcal{F}$. The result of substituting $y_{i}^{\prime}$ for $y_{i}$ and $u_{i j}^{\prime}$ for $u_{i j}$ in $C$ and its transform $C_{1}$ is the pair of formal power series $C^{\prime}$ and $C_{1}^{\prime}$ with coefficients in $G$. Let $C_{1}^{\prime \prime}$ be the expansion which results from $C_{1}$ upon making the substitution of $y_{i}^{\prime \prime}$ for $y_{i}, i \geqq 1$, and $u_{i j}^{\prime \prime}$ for $u_{i j}$. Evidently $C_{1}^{\prime \prime}$ can be constructed from $C^{\prime}$ by replacing $z_{i}$ by $z_{i+1}, 0 \leqq i<r, v_{i j}$ by $v_{i, j+1}$, and each coefficient of the expansion by its transform. $C_{1}^{\prime}$ may now be obtained from $C_{1}^{\prime \prime}$ by replacing $z_{r}$ by $z_{r}^{\prime}$. We see that, if $C^{\prime}$ vanishes identically, so does $C_{1}^{\prime \prime}$, and therefore $C_{1}^{\prime}$. It follows that the set of all polynomials 
which vanish when the $y_{i}$ and the $u_{i j}$ are replaced by the $y_{i}^{\prime}$ and $u_{i j}^{\prime}$ forms a difference ideal $\Psi$. Evidently $\Psi$ is prime and contains $A$.

Let $\Sigma$ be the set of all polynomials whose transforms of any order are in $\Psi$. Evidently $\Sigma$ is a prime reflexive difference ideal. We shall show that no polynomial of order less than $r$ in $y$, or free of $y$, holds $\Sigma$. First we consider a polynomial $D$ of order not exceeding $r-1$ in $y$ and show that it is not in $\Psi$. The substitutions $y_{i}=y_{i}^{\prime}, u_{i j}=u_{i j}^{\prime}$ become, in this special case, a linear transformation with an inverse. Since $D$ is not identically zero, the polynomial into which it is carried is not zero. Thus $D$ is not in $\Psi$. We now assume that all polynomials of effective order not exceeding $r-1$ and order not exceeding $r-1+k$ are not in $\Psi$. We shall show that a polynomial $D$ of order $r+k$ and effective order not exceeding $r-1$ in $y$ is not in $\Psi$. We have shown elsewhere ${ }^{6}$ that the resultant $R$ with respect to $y_{r+k}$ of $D$ and $A_{k}$ is a nonzero polynomial of order at most $r-1+k$ and effective order at most $r-1$. Our assumption shows that $R$ is not annulled by the substitutions in question, so that $D$ cannot be annulled by them. We can now show by induction that no polynomial of effective order at most $r-1$ in $y$ is in $\Psi$, so that no polynomial of order not exceeding $r-1$ in $y$ is in $\Sigma$. It follows that $\Sigma$ is one of the reflexive prime ideals whose solutions constitute the ordinary manifolds of $A$.

Some transform of any polynomial of $\Sigma$ must be annulled by the $y_{i}^{\prime}$ and $u_{i j}^{\prime}$. Now on substituting these quantities for the $y_{i}$ and $u_{i j}$ in the transform $C_{k}$ of a polynomial $C$ a term of zero degree is obtained which is equal to the result of substituting $\alpha$ for $y$ and $\gamma_{i}$ for $u_{i}$ in $C_{k}$. Since this term must vanish for every polynomial $C$ of $\Sigma$ and a suitable $k$ depending on $C, C_{k}$ and therefore $C$ is annulled by the general point of $\Lambda$. Then $\Sigma$ holds $\Lambda$. This proves the theorem.

4. Proof of the principal result. We now return to the proof of the possibility of the division of the essential irreducible manifolds of a difference polynomial into singular and ordinary manifolds. We consider an algebraically irreducible difference polynomial $A$ in unknowns $y_{1}, \cdots, y_{n}$ with coefficients in a difference field $\mathcal{F}$. Let $\mathfrak{M}$ be an essential irreducible manifold of $A$, and let $\Lambda$ be the corresponding reflexive prime ideal. We wish to show that $\mathfrak{M}$ is either an essential singular manifold of $A$ relative to each $y_{i}$ or an ordinary manifold relative to each $y_{i}$. For this purpose it will suffice to assume that $\mathfrak{M}$ is an essential singular manifold relative to $y_{1}$, and prove that it is singular relative to $y_{2}$.

${ }^{6}$ M. D. P., $\$ 14$. The presence of $u_{i j}$, which are not included in the discussion of M.D.P., does not affect the argument. 
If we make this assumption it follows that $\mathfrak{M}$ annuls $\partial A / \partial y_{1 r}$, where $r$ is the order of $A$ in $y_{1}$. Let $s$ be the order of $A$ in $y_{2}$. We form the resultant $R$ of $A$ and $\partial A / \partial y_{1 r}$, considered as algebraic polynomials in $y_{28}$. Since $A$ is irreducible, and cannot be a factor of $\partial A / \partial y_{1 r}$, $R$ is a nonzero polynomial, free of $y_{2 s}$, which is annulled by $\mathfrak{M}$. Since $R$ is of lower efiective order than $A$ in $y_{2}, \mathfrak{M}$ must be an essential singular manifold of $A$ relative to $y_{2}$. The proof is now complete.

RUTGERS UNIVERSITY

\section{DISTINCT REPRESENTATIVES OF SUBSETS}

MARSHALL HALL, JR.

1. Introduction. Let $W$ be a set of elements $a_{i}^{\prime} \cdot W=\left\{a_{1}, \cdots\right\}$ and let $U\left\{S_{1}, \cdots, S_{j}, \cdots\right\}$ be an indexed system of subsets of $W$. We wish to choose distinct representatives of the subsets. If $a_{j}=R\left(S_{j}\right)$ designates the representative of the subset $S_{j}$, then we require $R\left(S_{j}\right) \in S_{j}$ for all $j$ and $R\left(S_{j}\right) \neq R\left(S_{k}\right)$ if $j \neq k$. It is to be emphasized that subsets are distinguished only by their indices and distinct subsets may contain the same elements. An obviously necessary condition for the existence of distinct representatives is:

Condition C: Every $k$ distinct subsets contain between them at least $k$ distinct elements, for every finite $k$. P. Hall ${ }^{1}$ has shown that if the number of subsets is finite, condition $C$ is also sufficient for the existence of a system of distinct representatives, or SDR as we shall abbreviate. This condition is no longer sufficient if the number of subsets is infinite. As a counter example consider $U\left(S_{0}, S_{1}, \cdots\right\}$ where $S_{0}=\left\{a_{1}, a_{2}, \cdots\right\}, S_{i}=\left\{a_{i}\right\}, i=1,2, \cdots$. Here condition C is easily shown to hold for the subsets, but clearly no representative may be selected for $S_{0}$ which is not also a representative of some $S_{i}$.

In this paper it is shown that condition $C$ is sufficient if every subset $S_{j}$ is finite, and also an estimate on the number of systems of distinct representatives is given. This latter result is applied to Latin squares.

Theorem 1. Given an indexed system $U\left\{S_{1}, \cdots, S_{j}, \cdots\right\}$ of finite subsets of a set $W\left\{a_{1}, \cdots, a_{i}, \cdots\right\}$. If the subsets satisfy condi1947.

Received by the editors October 21,1947 , and, in revised form, November 8 ,

${ }^{1}$ P. Hall, On representatives of subsets, J. London Math. Soc. vol. 10 (1935) pp. 2630 . 